\title{
Research on Subsidence Intervention of Planet Rover Based on Wheel Soil-bin Test
}

\author{
Dawei Han ${ }^{1,2, a,{ }^{*}}$, Liangliang Ding ${ }^{1,2, b}$, Dianfu Liu ${ }^{1,2, c}$, Xiaoqing Yang ${ }^{1,2, d}$ and \\ Wenbo Feng ${ }^{1,2, e}$ \\ ${ }^{1}$ Shanghai Aerospace System Engineering Institute, Shanghai 201109, China \\ 2Shanghai Key Laboratory of Spacecraft Mechanism, Shanghai 201108, China \\ ahandawei021@163.com, b050410229@163.com, c13371995337@163.com, \\ dyangxiaoqingoo@126.com, e729661822@qq.com
}

Keywords: Planet rover, Subsidence estimation function, Wheel Soil-bin test, Subsidence intervention.

\begin{abstract}
It may encounter many unexpected events when the rover moving on the surface of the planet. Subsidence is fatal for the impact of mobile performance. It's very important to research on subsidence prediction and intervention measures when encountering unpredictable excessive subsidence. This paper linearized the function relationship between wheel and soil to establish simple equation for subsidence estimation. Then revise equation by means of wheel soil-bin test and establish subsidence estimation function used to estimate subsidence online. And use subsidence estimation function to study methods of Subsidence intervention, put forward two kinds of measures to solve the problem of subsidence.
\end{abstract}

\section{Introduction}

The concept of Planet rover subsidence is that under certain loading, the planet rover sinks through a variety of lunar rover Road, such as loose regolith, road slope, craters and so on. Research on planet rover subsidence is the one main content of crossing. It is also an important factor to affect the dynamic performance, maneuverability, ride comfort, energy consumption ratio and so on. This research plays an important role in the research of the planetary vehicle system.

\section{Overview of Research on Subsidence}

\subsection{The risk of Planet Rover Subsidence}

The LRV lunar rover carried on The "Appollo--16" spacecraft had a very high wheel slip phenomenon in the road. According to the astronauts' memories, the place was a little like a snow, or like a loose field. In some cases, the astronauts have to drag the LRV to the better terrain.

On 11th May 2005, the scientist of NASA declared that the rover "Opportunity" has stuck in soft sand on the Martian surface, which is likely to endanger its scientific mission. After nearly 5 weeks of hard trek, rover "Opportunity" has finally been lucky to escape the fate of the "death" on 3th June $2005^{[1]}$.

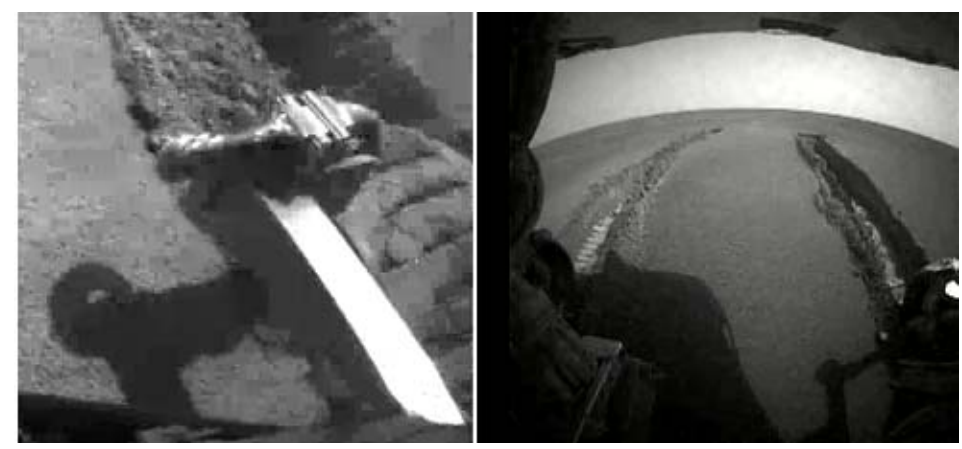

Fig.1 The picture of the opportunity rover sank in sand land 
In May 2009, "Courage" has experienced the same situation as the "opportunity". The wheels of "Courage" are stuck in the sand which caused the rover is hard to move. After almost 2 months later, "Courage" escaped from the place after various efforts and attempts by the researchers ${ }^{[2]}$.

China first lunar rover "YUTU" also encountered a similar excess subsidence situation in the process of lunar exploration mission (Fig. 2). Although "YUTU" did not encounter the extreme case of subsidence like "opportunity" and "courage” Rover", the problem of subsidence in the process of lunar exploration mission is still worth our vigilance.
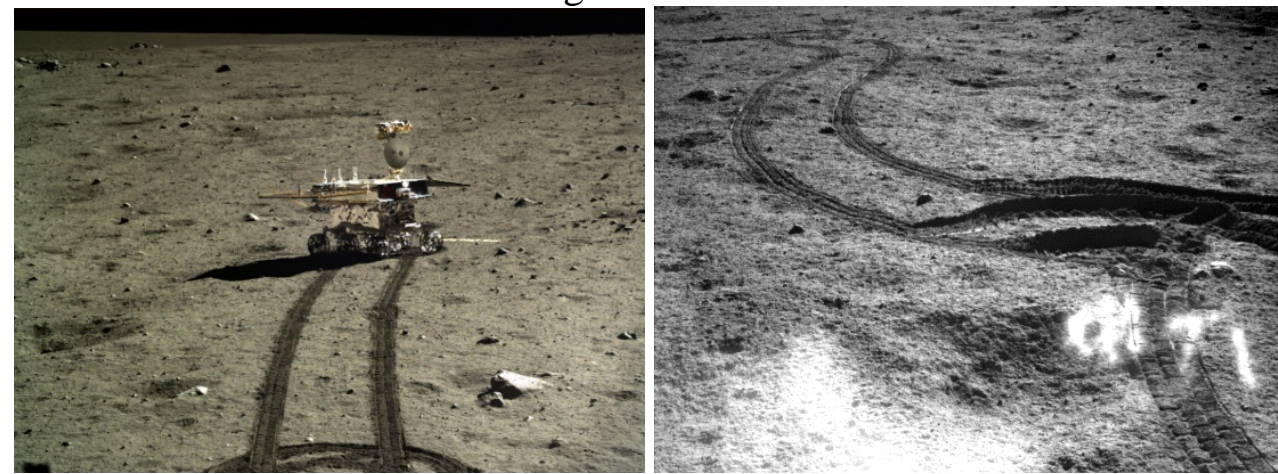

(a) The normal moving path

(b) the moving path in excess subsidence situation

Fig.2 The moving path of "YUTU" lunar rover

\section{Research on Subsidence}

At present, the researchers consider subsidence problem mainly from two angles: One is to prevent excessive subsidence through the intervention mechanism; the other is to study the remedial measures after the excessive subsidence situation.

\subsection{Research on Subsidence Monitoring System}

The research on how to monitor in real time or how to estimate the amount of subsidence during the vehicle moving is very limited. In the early stage of the study, researchers mainly got a wheel subsidence relative to the other wheel by providing high-precision sensors in the suspension joints. However, the absolute value of subsidence must be obtained by establishing the mathematical model. Therefore, some scholars proposed the concept that we can use the visual system (including stereo vision) to identify the relevant parameters to estimate the subsidence and soil parameters. In the actual process of applying, it was still restricted by the aspects of suspension \& vision systems, energy consumption, computing speed and so on.

In the study, the MIT Christopher A. Brooks, Karl D. Iagnemma, Steven Dubowsky et present a gray cameras and linearized soil interaction model to evaluate passage capacity of soil ${ }^{[3]}$. The test showed that the methods not only take into account both computing speed and data transfer volume, but also had a good adaptability for different terrain and lighting conditions, referring to Fig.3.

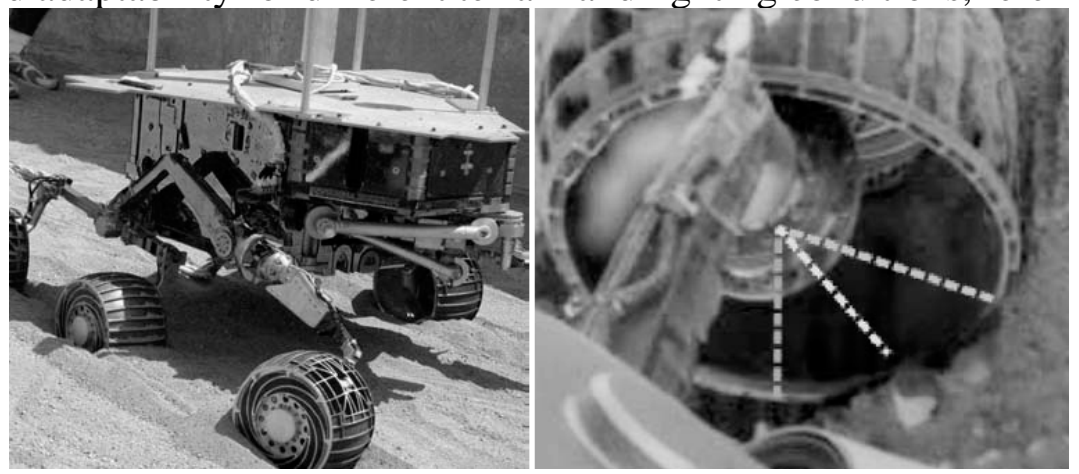

Fig.3 The visual identification system of subsidence

Taking into account the need for special equipment for soil parameters online estimation is very inconvenient, researchers studied only the vehicle itself. For example, MIT Shinwoo kang who used the related capabilities of the vehicle itself established a computational model for Soil cohesion $c$ and inner friction angel $\varphi$ of the soil. This method is used to determine the relationship between the 
driving torque and the relative settlement. Compared with the experimental results, the model result is very close to it ${ }^{[4]}$.

A common feature of these studies is that researcher didn't think the subsidence amount is the only indicator to judge passage capability. Subsidence amount can't reflect passage capability of vehicle. We need to consider the driving force (torque) together with subsidence, so that we can assess the safety of the vehicle.

\subsection{Research on the Remedial Measures for Excessive Settlement}

US "Opportunity" rover encountered excessive subsidence problem which require the researcher do the remedy in the excessive subsidence situation. Fortunately, US rover project made 4 rovers so that they had the opportunity to work on the ground simulation to carry out remedial measures.

In the "Spirit" rover announcement, MER project manager, JPL John Callas, led his team to do the remedy work for excessive subsidence. In this case, they think the rover was in the case of excessive subsidence on the slope. They simulated the situation in the laboratory (Fig. 4). JPL rover team got the possible way to pass through the subsidence situation by wheel drive and slewing gear according to specific sequence movement. However, dealing with the problem of excessive settlement is very complicated and difficult.

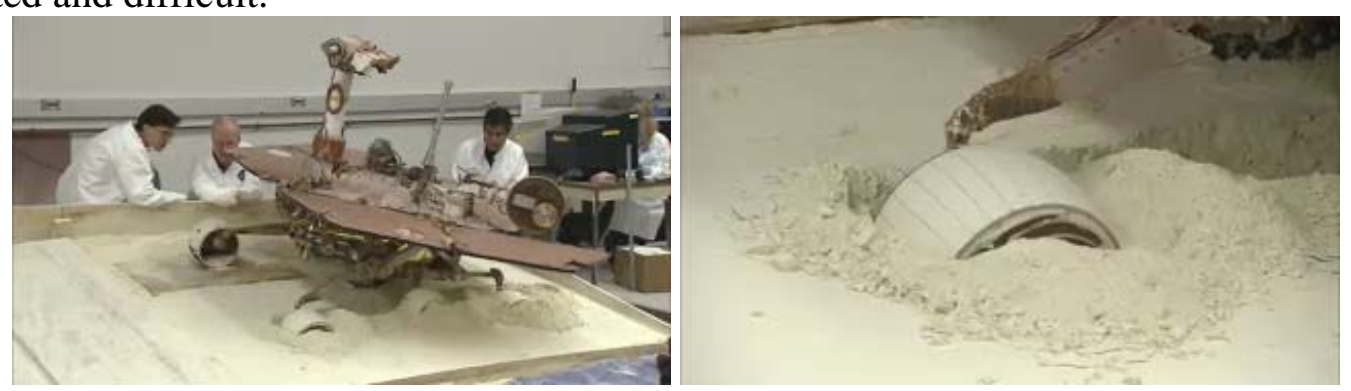

Fig.4 The simulation of subsidence dangerous state of the spirit rover on Mars

By all accounts, planet rover that drives in the planet surface may encounter a lot of unexpected events, and the influence of subsidence on the moving performance is fatal. It is very important to study the subsidence prediction and interventions in the event of unforeseen terrain that can produce excessive subsidence.

\section{Main Contents of the Research on Subsidence}

The biggest challenge of the planet rover driving comes from pilotless. This requires planet vehicle mobile subsystem to supply more driving parameter in the case of a limited sensor configuration and the minimum energy consumption. For all the parameters, important parameters from the ground and the ground and the wheel force - drawbar pull is difficult to obtain. Though the ground wheel bench tests, large hook traction means that the vehicle's performance is good, while small or negative hook traction means the planet is not easy to get through and easily caught in the car, which may cause excessive subsidence. Therefore, the core of the subsidence problem is to establish the relationship between the traction force and the hook.

On the other hand, although the amount of subsidence can be obtained through an external sensor, the current inspection of the detector cannot be equipped with additional sensors to get the amount of subsidence. It is also known by the ground wheel bench test that if the terrain parameters, the driving states, the traction force, the driving torque and the load are known, the amount of subsidence can be estimated by the mechanical calculation.

Also, it may have risk for planet rover to identify the excess. Off-line method is often used in some subsidence measurement or estimation that was developed by researchers. Planet rover carry out a lot of walking test in a region according to a certain step, then the sensor record the date and send the data to the ground to do the calculation. This method requires the planet to travel in a dangerous area of time; also it may encounter excessive subsidence in the process of calculation.

By all accounts, the core of planet rover subsidence research is not how to measure subsidence, but requires planet rover to carry out the estimation of traction and subsidence in real time during 
working, or to judge the subsidence amount through the change of the hook traction. In summary, the main content of the research on the subsidence is that through establishing a simple relationship between the traction force and subsidence amount to meet the requirements of real-time measurement of the planet surface.

In this paper, we will first make the linear derivation of wheel-terrain interaction to establish an intuitive and simple equation for the subsidence estimation. Then, we will verify and modify the equation by using the data of bench test to establish the subsidence measurement program which can be used for on-line estimation.

\section{Study on the Relationship between Wheel-Terrain Interaction and Subsidence}

According to the theory of ground mechanics, the traction force $D P$ and the wheel load $W$ based on the stress distribution under the wheel can be calculated as below ${ }^{[5]}$ :

$$
\begin{aligned}
& D P=r b \int_{0}^{\theta_{1}}[\tau(\theta) \cos \theta-\sigma(\theta) \sin \theta] d \theta \\
& W=r b \int_{0}^{\theta_{1}}[\tau(\theta) \sin \theta+\sigma(\theta) \cos \theta] d \theta
\end{aligned}
$$

In the equation, $r$ is wheel radius; $b$ is wheel width; $\sigma(\theta)$ is normal stress between the wheel bottom and any point $\mathrm{P}$ in contract area(With the ground vertical angle $\theta$ ); ${ }^{\tau(\theta)}$ is the shear stress of the wheel at that point; $\theta_{1}$ is the asymptotic angle of the wheel.

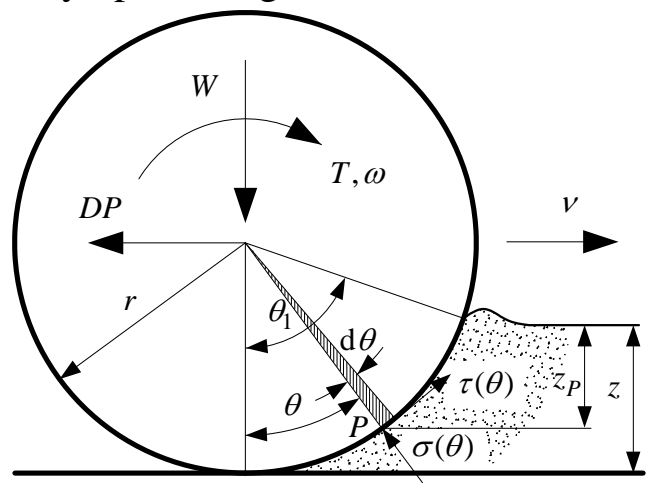

Fig.5 The force diagram of wheel rolling

In Eq. 1, we can prove that $\sigma$ and $\tau$ can be calculated by estimating the lunar soil parameter $c$ and internal friction $\varphi$ by linearization, and then calculate traction force $D P$. This method requires the measurement of load, torque, subsidence and slip rate, which can be measured in theory. However, this method is difficult to use in the situation of actual walking.

For the analysis of the influence factors of the hook traction, soil characteristics, wheel properties and control condition may impact a lot. Once these influencing factors are determined, all kinds force and torque of wheel-terrain interaction and sinkage can be obtained by calculating. And the driving torque $T$, sinkage $z$ and hook traction $D P$ is each function of soil characteristics, wheel properties and operating conditions. It can be envisaged that if the driving torque and subsidence amount is determined, the traction force can be calculated.

In order to establish the above relationship, we can make alignment of Eq. 1 . Let $\theta_{m}$ be the maximum positive stress $\sigma_{\max }$ and the maximum shear stress $\tau_{\max }$ in the contact area of the bottom of the wheel, while $0 \leq \theta \leq \theta_{m}$, then,

$$
\sigma_{1}(\theta)=\frac{2 \theta}{\theta_{1}} \sigma_{\max }, \quad \tau_{1}(\theta)=\frac{2 \theta}{\theta_{1}} \tau_{\max }
$$

While $\theta_{m} \leq \theta \leq \theta_{1}$, then:

$$
\sigma_{2}(\theta)=\frac{2\left(\theta_{1}-\theta\right)}{\theta_{1}} \sigma_{\max }, \quad \tau_{2}(\theta)=\frac{2\left(\theta_{1}-\theta\right)}{\theta_{1}} \tau_{\max }
$$

Put Eq. 3 and 4 into Eq. 1, then: 


$$
\begin{aligned}
D P & =r b \int_{0}^{\theta_{m}}\left[\tau_{1}(\theta) \cos \theta-\sigma_{1}(\theta) \sin \theta\right] d \theta+r b \int_{\theta_{m}}^{\theta_{1}}\left[\tau_{2}(\theta) \cos \theta-\sigma_{2}(\theta) \sin \theta\right] d \theta \\
& =\frac{2 r b}{\theta_{1}}\left(\left(2 \cos \frac{\theta_{1}}{2}-\cos \theta_{1}-1\right) \tau_{\max }-\left(2 \sin \frac{\theta_{1}}{2}-\sin \theta_{1}\right) \sigma_{\max }\right)
\end{aligned}
$$

$\tau_{\max }$ and $\sigma_{\max }$ can be calculated by driving torque $T$ and vehicle load $W$ :

$T=r b \int_{0}^{\theta_{1}} \tau(\theta) d \theta=\frac{r^{2} b \theta_{1} \tau_{\max }}{2}$

Then:

$$
\begin{aligned}
\tau_{\max } & =\frac{2 T}{r^{2} b \theta_{1}} \\
W & =r b \int_{0}^{\theta_{1}}(\sigma(\theta) \cos \theta+\tau(\theta) \sin \theta) d \theta \\
& =\frac{2 r b}{\theta_{1}}\left(\left(2 \cos \frac{\theta_{1}}{2}-\cos \theta_{1}-1\right) \sigma_{\max }+\left(2 \sin \frac{\theta_{1}}{2}-\sin \theta_{1}\right) \tau_{\max }\right)
\end{aligned}
$$

Then:

$$
\sigma_{\max }=\frac{\frac{\theta_{1}}{2 r b} W-\left(2 \sin \frac{\theta_{1}}{2}-\sin \theta_{1}\right) \tau_{\max }}{2 \cos \frac{\theta_{1}}{2}-\cos \theta_{1}-1}=\frac{\frac{\theta_{1}}{2 r b} W-\left(2 \sin \frac{\theta_{1}}{2}-\sin \theta_{1}\right) \frac{2 T}{r^{2} b \theta_{1}}}{2 \cos \frac{\theta_{1}}{2}-\cos \theta_{1}-1}
$$

Put Eq. 6 and 7 into Eq. 5, then:

$$
D P=-\frac{\left(2 \sin \frac{\theta_{1}}{2}-\sin \theta_{1}\right)}{\left(2 \cos \frac{\theta_{1}}{2}-\cos \theta_{1}-1\right)} W+\frac{\left(2 \cos \frac{\theta_{1}}{2}-\cos \theta_{1}-1\right)^{2}+\left(2 \sin \frac{\theta_{1}}{2}-\sin \theta_{1}\right)^{2}}{\left(2 \cos \frac{\theta_{1}}{2}-\cos \theta_{1}-1\right)} \frac{4}{\theta_{1}^{2}} \frac{T}{r}
$$

Due to $\theta_{1}=\cos ^{-1}(1-z / r)$ always less than $60^{\circ}(0 \leq z \leq r / 2)$

Them $2 \sin \left(\theta_{1} / 2\right)-\sin \theta_{1} \approx \theta_{1}^{3} / 8, \quad 2 \cos \left(\theta_{1} / 2\right)-\cos \theta_{1}-1 \approx \theta_{1}^{2} / 4$, so Eq. 8 can be simplified as:

$$
D P=-\frac{\theta_{1}}{2} W+\left(1+\frac{\theta_{1}^{2}}{4}\right) \frac{T}{r} \approx \frac{T}{r}-\frac{\theta_{1}}{2} W
$$

Since $\theta_{1} \approx 2 z / r$, Eq. 9 can be transformed as:

$$
\frac{D P}{W} \approx \frac{T}{r W}-\frac{z}{r}
$$

Through the above derivation, the Eq. 10 represents the functional relation between four parameters: traction force $D P$, driving torque $T$, $\operatorname{load}^{W}$ and subsidence $Z$. This relationship is very simple and the real time of rover online performance estimation can be guaranteed. In order to discuss the method, we call the Eq. 10 is subsidence estimation function.

\section{Subsidence Estimation Modification based on Wheel Soil-Bin Test}

We take the wheel soil-bin test of "YUTU" rover as an example to verify the validity of Eq. 10 (Fig. 6). Under the load of $3 \mathrm{~kg}, 7 \mathrm{~kg}, 10 \mathrm{~kg}, 15 \mathrm{~kg}$ and $20 \mathrm{~kg}$, we obtained experimental data through wheel driving on the median grain size $70 \mu \mathrm{m}$ simulated soil. Then we verified and modified the subsidence estimation function of section 3 . 

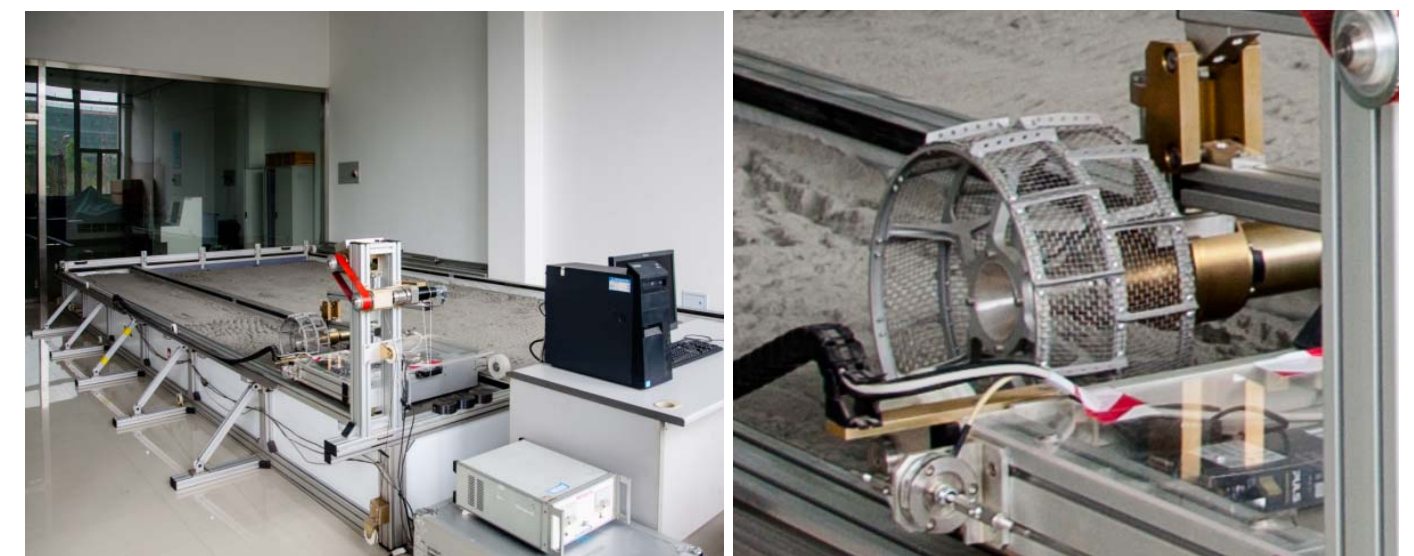

(a) The device of the wheel soil-bin test(b) The wheel soil-bin test of "YUTU" rover Fig.6 The wheel soil-bin test

We use Eq. 10 to fit the relation of the subsidence estimation function under the load of $3 \mathrm{Kg}$. In the fitting process, it needs to introduce correction factor to make the fitting error minimum. The test data of "YUTU" rover under $3 \mathrm{~kg}$ vertical load is shown as below table.

Table 1 . The experimental date of wheel soil-bin test at $3 \mathrm{~kg}$ vertical load

\begin{tabular}{c|c|c|c|c|c}
\hline & Slip Rate & $\begin{array}{c}\text { Sinking } \\
\text { coefficient }\end{array}$ & $\begin{array}{c}\text { Drive } \\
\text { Coefficient }\end{array}$ & $\begin{array}{c}\text { Traction force coefficient } \\
K_{D P}\end{array}$ & $\begin{array}{c}\text { Fitted value of Eq. 10 } \\
K_{D P}^{\prime}\end{array}$ \\
\cline { 2 - 6 } & SLIP & $z / r$ & $T /(r W)$ & $D P / W$ & $T /(r W)-z / r$ \\
\hline 1 & 0.060 & 0.076 & 0.197 & -0.051 & 0.121 \\
\hline 2 & 0.100 & 0.078 & 0.354 & 0.104 & 0.276 \\
\hline 3 & 0.140 & 0.082 & 0.483 & 0.232 & 0.400 \\
\hline 4 & 0.180 & 0.090 & 0.561 & 0.290 & 0.472 \\
\hline 5 & 0.220 & 0.097 & 0.629 & 0.327 & 0.532 \\
\hline 6 & 0.260 & 0.106 & 0.663 & 0.388 & 0.591 \\
\hline 7 & 0.300 & 0.113 & 0.704 & 0.392 & 0.618 \\
\hline 8 & 0.340 & 0.122 & 0.740 & 0.397 & 0.613 \\
\hline 9 & 0.380 & 0.131 & 0.744 & 0.457 & 0.633 \\
\hline 10 & 0.420 & 0.138 & 0.771 & 0.445 & 0.652 \\
\hline 11 & 0.460 & 0.146 & 0.798 & 0.463 & 0.653 \\
\hline 12 & 0.500 & 0.152 & 0.805 & 0.467 & 0.669 \\
\hline 13 & 0.540 & 0.156 & 0.824 & 0.490 & 0.666 \\
\hline 14 & 0.580 & 0.164 & 0.830 & 0.524 & 0.651 \\
\hline 15 & 0.620 & 0.175 & 0.826 & 0.522 & 0.646 \\
\hline 16 & 0.660 & 0.186 & 0.833 & 0.526 & \\
\hline
\end{tabular}

According to slip rate change trend, we can draw curves using traction force factor $K_{D P}$ of table 1 and fitted value of Eq. 10. As shown in Fig. 7, we can see that the change trend is consistent with the slip rate $0 \sim 0.6$.

It can be clearly seen from Fig. 7 that the estimation function Eq. 10 has a significant correlation with the test results. In order to obtain the correction value, the Eq. 10 is rewritten as follows:

$$
\frac{D P}{W} \approx C_{1} \frac{T}{r W}+C_{2} \frac{z}{r}+C_{3}
$$




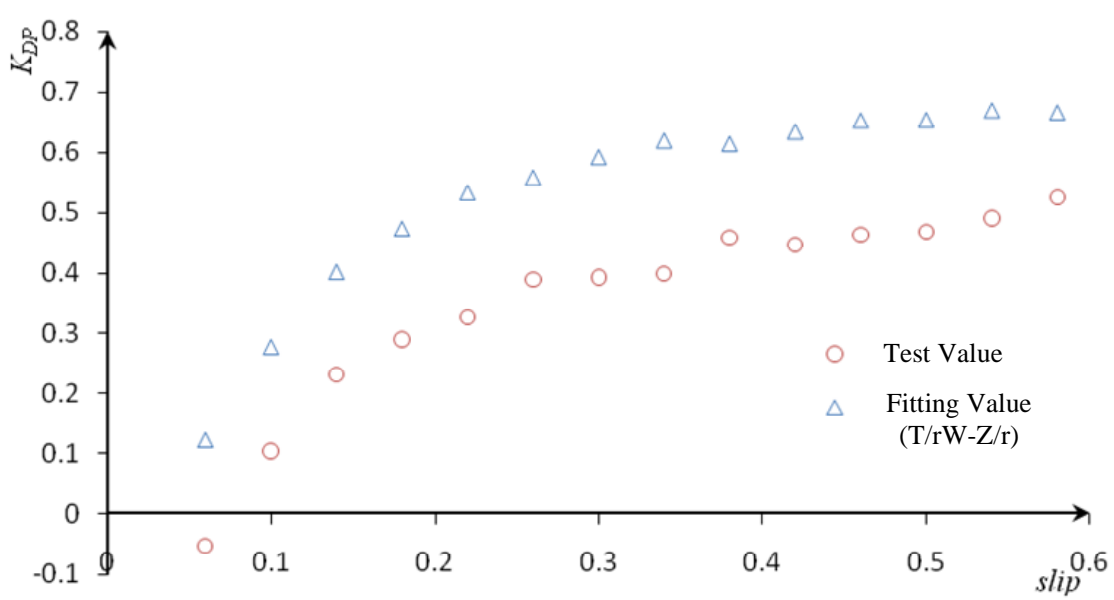

Fig.7 The contrast curves of test value and fitted value at $3 \mathrm{~kg}$ vertical load In order to obtain the parameters $C_{1} 、 C_{2} 、 C_{3}$, Eq. 11 can be rewritten as a matrix form: $A \cdot\left[\begin{array}{l}C_{1} \\ C_{2} \\ C_{3}\end{array}\right]=B$

As former,

$A=\left[\begin{array}{ccc}\left(\frac{T}{r W}\right)_{1} & \left(\frac{Z}{r}\right)_{1} & 1 \\ \cdots & \cdots & \cdots \\ \left(\frac{T}{r W}\right)_{n} & \left(\frac{Z}{r}\right)_{n} & 1\end{array}\right], \quad B=\left[\begin{array}{c}\left(\frac{D P}{W}\right)_{1} \\ \vdots \\ \left(\frac{D P}{W}\right)_{n}\end{array}\right]$

In order to calculate $C_{1} 、 C_{2} 、 C_{3}$, then:

$$
\left[\begin{array}{l}
C_{1} \\
C_{2} \\
C_{3}
\end{array}\right]=\left(A^{T} A\right)^{-1} \cdot A^{T} B
$$

Put the data of table1 into Eq. 13 to obtain the correction term of three parameters $\left[\begin{array}{c}C_{1} \\ C_{2} \\ C_{3}\end{array}\right] \approx\left[\begin{array}{c}1 \\ -1 \\ 0.2\end{array}\right]$, we can see the result as below:

$$
\frac{D P}{W} \approx \frac{T}{r W}-\frac{Z}{r}-0.2
$$

The modified fitted values are compared with the experimental values shown in Fig. 8: 


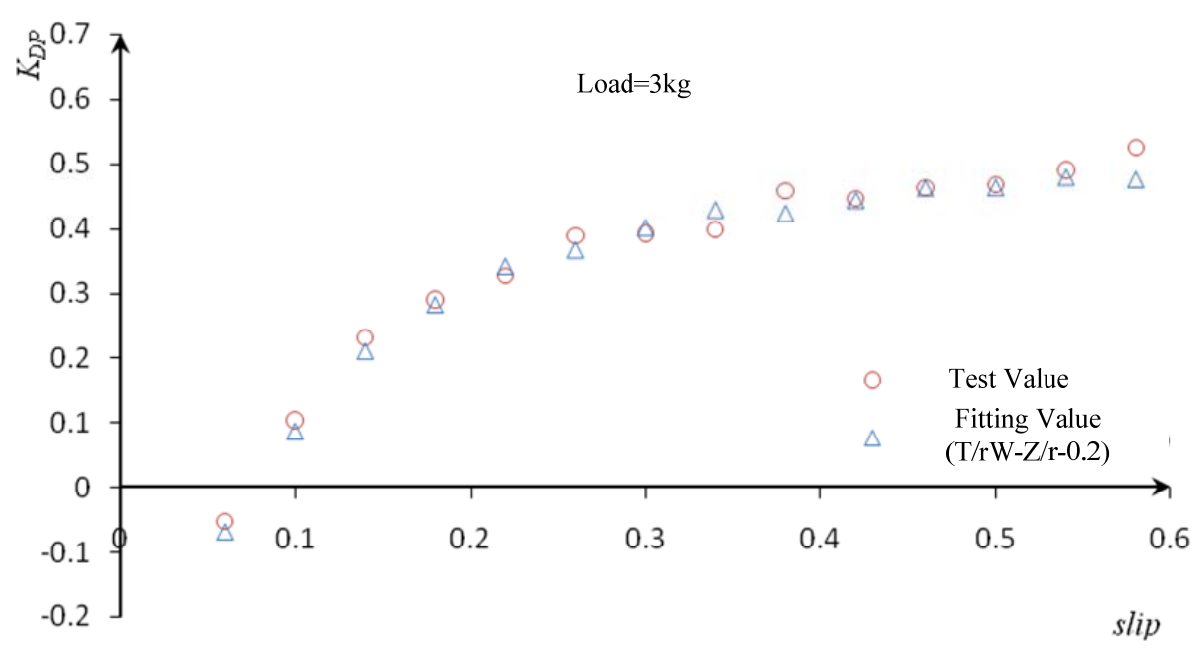

Fig.8 The contrast curves of test value and fitted value corrected

Similar as $3 \mathrm{~kg}$ vertical load, the data also need to be amended accordingly under the other vertical load $(7,10,15,20)$. In order to simplify the space, there is no longer to discuss the specific revise process. The revised results of each load are as table 2.

Table 2. The subsidence estimation function corrected at all loads

\begin{tabular}{c|c|c}
\hline Number & Load & Correction of subsidence estimation function \\
\hline 1 & $3 \mathrm{~kg}$ & $\frac{D P}{W} \approx \frac{T}{r W}-\frac{Z}{r}-0.2$ \\
\hline 2 & $7 \mathrm{~kg}$ & $\frac{D P}{W} \approx \frac{T}{r W}-\frac{Z}{r}-0.15$ \\
\hline 3 & $10 \mathrm{~kg}$ & $\frac{D P}{W} \approx \frac{T}{r W}-\frac{Z}{r}-0.13$ \\
\hline 4 & $15 \mathrm{~kg}$ & $\frac{D P}{W} \approx \frac{T}{r W}-\frac{Z}{r}-0.13$ \\
\hline 5 & $20 \mathrm{~kg}$ & $\frac{D P}{W} \approx \frac{T}{r W}-\frac{Z}{r}-0.13$ \\
\hline
\end{tabular}


The load correction curves are shown in Fig. 9.

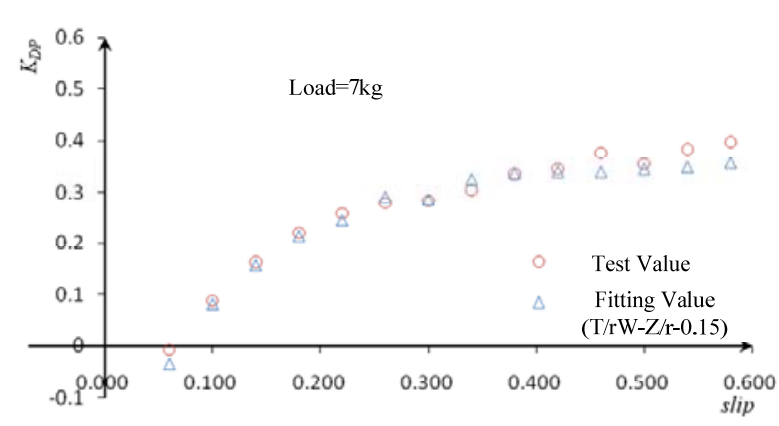

(a) $7 \mathrm{~kg}$ vertical load

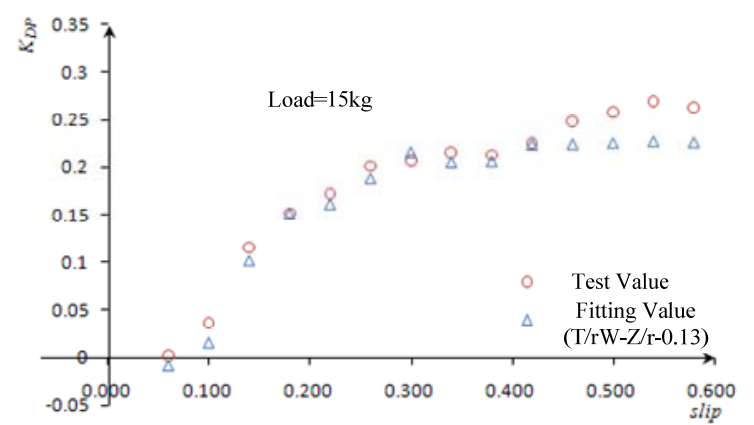

(c) $15 \mathrm{~kg}$ vertical load

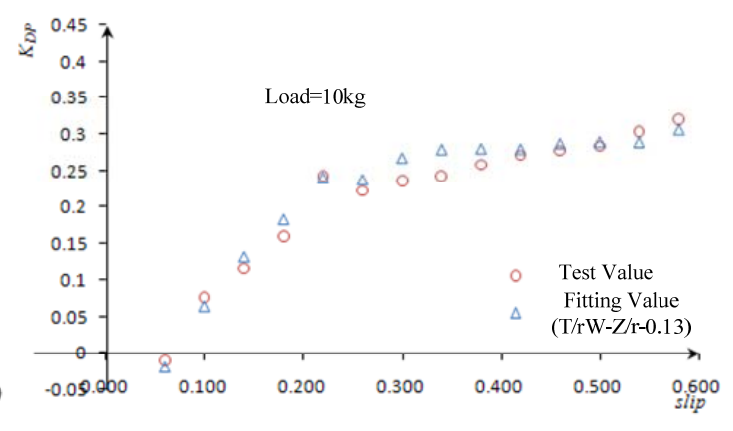

(b) $10 \mathrm{~kg}$ vertical load

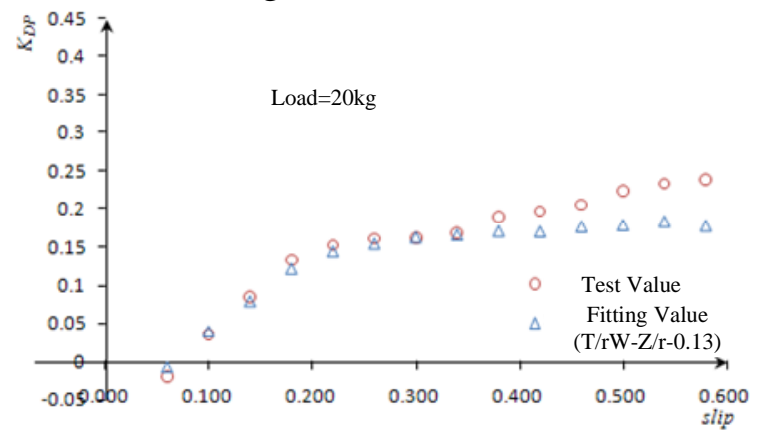

(d) $20 \mathrm{~kg}$ vertical load

Fig. 9 The contrast curves of test value and fitted value corrected at else loads

The results show that according to the increase of load, the trend of this correction factor is reduced between correction factor $C_{3}$ 0.13 0.20 under various loading conditions. The correction factor can be adopted in the 0.13 or so for simplify, which can achieve a satisfactory result.

Therefore, the subsidence estimation function can be written as follows:

$$
\frac{D P}{W} \approx \frac{T}{r W}-\frac{z}{r}-0.13
$$

\section{Use Subsidence Estimation Function to Intervene}

Through Eq. 15, we can analyze the relationship between terrain track and traction force $D P$, wheel torque $T$ and subsidence $z$ quantitatively. The proper threshold value can be set up according to the task or the environment factors. According to Eq. 15, only the below information was known, we can carry out real time measurement: subsidence estimated function (including the correction factor) of hook traction, driving torque and subsidence; load on wheel during driving; driving torque on the wheel during driving; traction force.

Based on the theoretical analysis, subsidence estimation function can be established by the test of wheel platment in the above mentioned paramenters. Using vehicle telemetry paramenters, we can easily get the wheel load and driving torque. The wheel load can be obtained according to the position and the force analysis of the inspection device, and the driving torque can be converted according to the measured motor current. From the point of view of the relationship between the traction coefficient and the slip ratio in the test of wheel platform, the hook traction of the rover changes a little in the case of high slip ratio (i.e., the occurrence of large sink probability)(Fig. 10). Under the known soil paraments and load, the hook traction value in the case of high slip ratio can be obtained through wheel bench test.

According to the above analysis, it is known that the key technologies to prevent subsidence need to break through are: study on the relationship between traction force, driving force and subsidence; research on load estimation of wheel based on Vehicle kinematics and dynamic model; research on the relationship between motor current and driving torque; research on the method of estimating the traction force based on the behavior of the rover. 


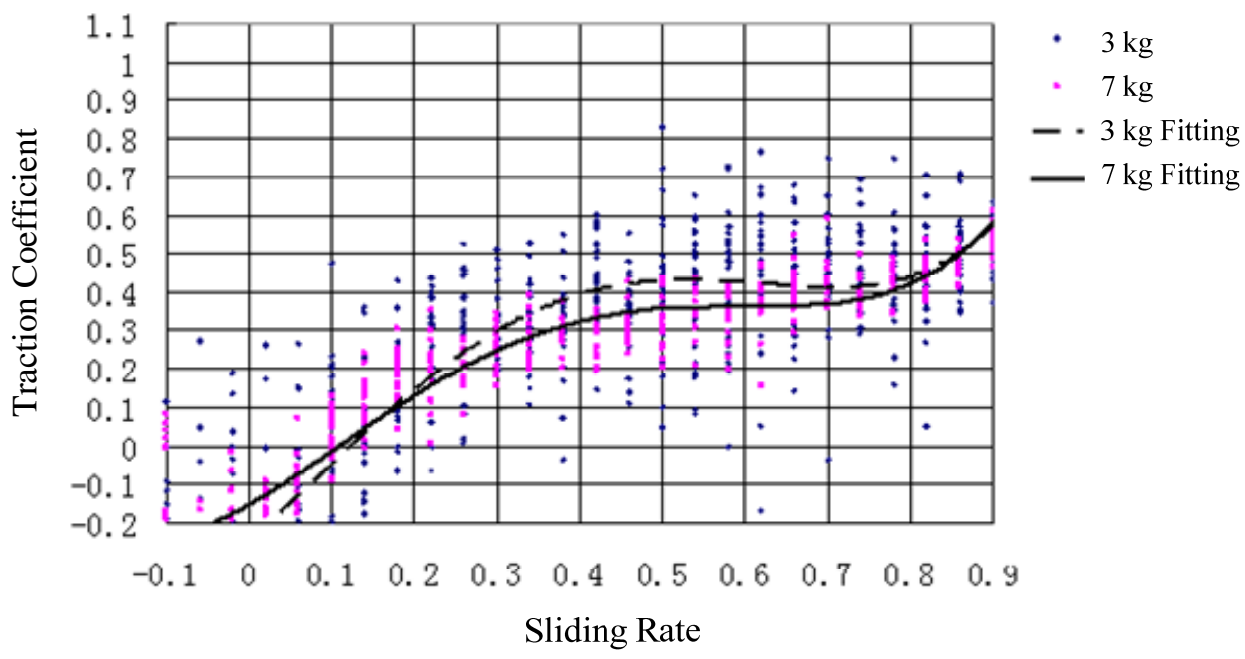

Fig.10 The curve of traction coefficient and sliding rate

From the results of the soil-bin test, the hook traction force has little change in the case of a large slip rate, so the maximum value of the traction can be initially estimated from the soil condition. The maximum drive current value of the star car is calculated by the subsidence estimation function of Eq. 15 , which is convenient for the interpretation of the on-orbit data. When the driving current of planet rover is close to the calculated maximum drive current value, there is the risk of excessive subsidence, immediately let the planet rover to stop the movement, re-planning the movement of the planet's trajectory to avoid the sinking of the rover.

In the event of planet rover subsidence, the maximum amount of wheel can also be estimated from Eq. 15 from the maximum value of the current telemetry to facilitate ground simulation. In the process of ground simulation (Fig. 11), found that due to the combination of wheel slip and pawl, the soil back of the wheel contact area turns to soil package, and the soil front of the wheel contact area turns to collapse area. Therefore, we can take the alternating method of wheel reversing, so that the soil can be continuously transported to the bottom of the wheel, and the amount of subsidence gradually reduced. This method can effectively help the planet rover leave off the storm.
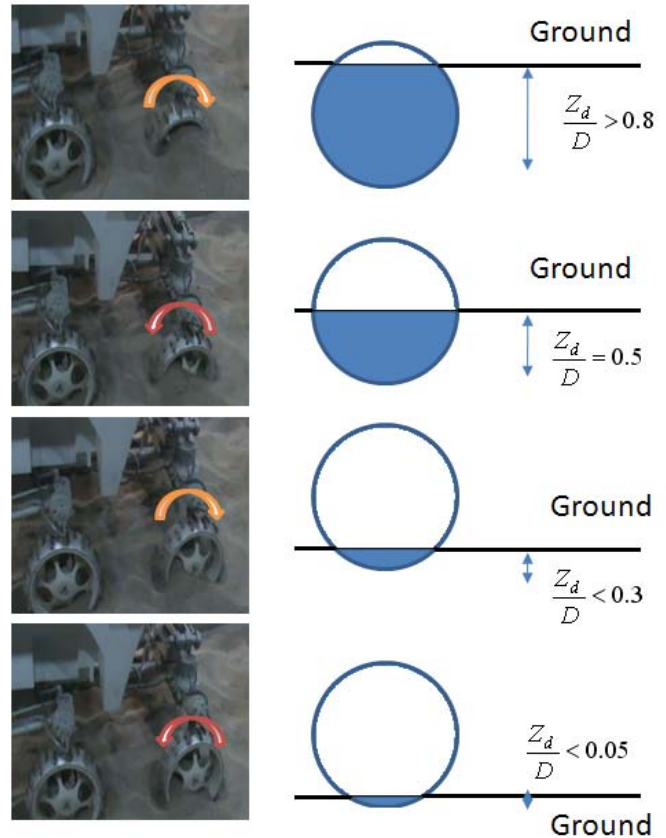

Fig.11 The test of planet rover bailed out sinking by means of ground-based simulation 


\section{Summary}

Based on the problem of subsidence at home and abroad, this paper puts forward that the problem of subsidence should proceed from the two aspects of prevention and over-subsidence emergency rescue. From the single-wheel bench test and the vehicle test, under the current test conditions, the following conclusions are credible: based on the linear analysis of the soil interaction model and the results of the bench test, the estimation function of the dome can be obtained. The function is perceptual intuition and simple, and it does not need to identify the soil mechanics performance parameters online. The function will have a wide range of applicability. The subsidence estimation function makes subsidence interventions easy. The maximum drive current value of the star car can be calculated by the subsidence estimation function, which can be used to evaluate the on-orbit data. In the event of a wheel sinking, the maximum amount of wheel subsidence can also be estimated from the maximum value of the current telemetry, which facilitates ground simulation.

\section{Acknowledgements}

This research was financially supported by Science and Technology Commission of Shanghai Municipality, project number 12170700500.

\section{References}

[1]. E.T. Baumgartner, J.J. Biesiadecki, R.G. Bonitz, Mars Exploration Rover Surface Operations: Driving Opportunity at Meridiani Planum, IEEE. (2005)

[2]. P. Chris Leger, W. Trebi-Ollennu, Mars Exploration Rover Surface Operations: Driving Spirit at Gusev Crater. (2005)

[3]. I. Halatci, C.A. Brooks, K. Iagnemma, Terrain Classification and Classifier Fusion for Planetary Exploration Rovers, IEEE Aerospace Conference Proceedings. (2007).

[4]. K. Iagnemma, S. Kang, H. Shibly, S. Dubowsky, Online terrain parameter estimation for wheeled mobile robots with application to planetary rovers, IEEE Transactions on Robotics. (2004)

[5]. M.G. Bekker, Theory of land locomotion - The mechanics of vehicle mobility, The university of Michigan Press, Ann Arbor, Michigan.(1956) 